\title{
Assessing Adherence to Antihypertensive Medication by Means of Dose-Dependent Reference Plasma Concentration Ranges and Ultra-High Performance Liquid Chromatography-Ion Trap Mass Spectrometry Analysis
}

\author{
Lea Wagmann ${ }^{1}$, Aline C. Vollmer ${ }^{1}$, Lucas Lauder ${ }^{2}\left(\mathbb{D}\right.$, Felix Mahfoud ${ }^{2,3}{ }^{(D}$ and Markus R. Meyer ${ }^{1, *}$ \\ 1 Center for Molecular Signaling (PZMS), Institute of Experimental and Clinical Pharmacology and Toxicology, \\ Department of Experimental and Clinical Toxicology, Saarland University, 66421 Homburg, Germany; \\ lea.wagmann@uks.eu (L.W.); aline.vollmer@uks.eu (A.C.V.) \\ 2 Klinik für Innere Medizin III, Kardiologie, Angiologie und Internistische Intensivmedizin, \\ Universitätsklinikum des Saarlandes, Saarland University, 66421 Homburg, Germany; \\ lucas.lauder@uks.eu (L.L.); felix.mahfoud@uks.eu (F.M.) \\ 3 Institute for Medical Engineering and Science, MIT, Cambridge, MA 02142, USA \\ * Correspondence: m.r.meyer@mx.uni-saarland.de
}

Citation: Wagmann, L.; Vollmer, A.C.; Lauder, L.; Mahfoud, F.; Meyer, M.R. Assessing Adherence to Antihypertensive Medication by Means of Dose-Dependent Reference Plasma Concentration Ranges and Ultra-High Performance Liquid Chromatography-Ion Trap Mass Spectrometry Analysis. Molecules 2021, 26, 1495. https://doi.org/ $10.3390 /$ molecules26051495

Academic Editor: Roberto Mandrioli

Received: 23 February 2021

Accepted: 5 March 2021

Published: 9 March 2021

Publisher's Note: MDPI stays neutral with regard to jurisdictional claims in published maps and institutional affiliations.

Copyright: (c) 2021 by the authors. Licensee MDPI, Basel, Switzerland. This article is an open access article distributed under the terms and conditions of the Creative Commons Attribution (CC BY) license (https:// creativecommons.org/licenses/by/ $4.0 /)$.

\begin{abstract}
Poor adherence to antihypertensive drug therapy is a well-recognized problem and can be assessed by mass spectrometry-based analyses of body fluids. However, contrary statements exist whether drug quantification in blood or qualitative screening in urine is more suitable. The present pilot study aimed to further elucidate the power of blood plasma drug concentrations for adherence monitoring by developing and validating a quantification procedure for nine antihypertensive drugs (amlodipine, bisoprolol, candesartan, canrenone, carvedilol, metoprolol, olmesartan, torasemide, and valsartan) in blood plasma using liquid-liquid extraction and an ultra-high-performance liquid chromatography-ion trap mass spectrometry analysis. The procedure should then be used for an adherence assessment and compared with the results of an established qualitative urine screening. Selectivity, carryover, matrix effect, accuracy, precision, dilution integrity, and stability were successfully validated, except for amlodipine. The applicability was demonstrated by analyzing 19 plasma samples containing 28 antihypertensive drugs and comparing the measured concentrations with calculated dose-dependent reference plasma concentration ranges. The interpretation of plasma concentrations was found to be more sophisticated and time-consuming than that of urine screening results, and adherence could not be assessed in two cases (10\%) due to measured plasma concentrations below the lower limit of quantification. However, 14 out of 19 subjects were classified as adherent $(75 \%)$ and three as nonadherent (15\%), in contrast to $19(100 \%)$ that were claimed to be adherent based on the results of the qualitative urine screening. Nevertheless, further data is needed to estimate whether plasma quantification is superior in terms of assessing adherence to antihypertensive medication.
\end{abstract}

Keywords: hypertension; antihypertensive drugs; bioanalysis; adherence monitoring; LC-MS/MS

\section{Introduction}

Hypertension remains one of the most prevalent cardiovascular risk factors leading to more than nine million premature deaths each year [1]. Blood pressure lowering has been shown to reduce the risk of major cardiovascular disease events, such as stroke, heart failure, coronary heart disease, and all-cause mortality [2,3]. The current guidelines recommend the administration of antihypertensive drugs such as beta-blockers, calcium antagonists, diuretics, angiotensin converting enzyme inhibitors, or angiotensin receptor blockers (ARBs) [3,4], preferably in fixed-dose combinations. 
According to the WHO, adherence is "the extent to which a person's behavior [ ... ] corresponds with agreed recommendations from a health care provider", and adherence to prescribed medication poses an essential part [5]. Poor adherence to antihypertensive medication is frequently observed in patients with uncontrolled blood pressure and might be an underlying cause for hypertensive urgencies [6-8] but can also be mistaken for treatment resistance [9]. Numerous studies have reported that about half of hypertensive patients do not take their blood pressure-lowering medications as prescribed [9-12]. Hence, the assessment of patients' adherence to antihypertensive medication is becoming an increasingly recognized component of successful hypertension management [5]. These measures include interviews, diaries, or questionnaires that are easily accessible, fast, and inexpensive but were shown to overestimate the adherence $[13,14]$. The counting of remaining dosage units such as tablets or the collection of refill data represent objective strategies but have limited reliability [6]. Electronic monitoring devices, so-called medication event monitoring systems that record the time and date when a medication container was opened, are a recent innovation in this field, but the expense of these devices precludes their widespread use and opening them does not necessarily prove any intake [5]. Bioanalytical methods, which may be considered as the gold standard [6], allow the unambiguous detection of drugs and/or their metabolites in body fluids and, thus, a proof an intake. Hence, they directly and objectively assess patients' adherence. Such methods are of increasing importance due to the high specificity and sensitivity of, particularly, liquid chromatography (LC) coupled to low-resolution or high-resolution mass spectrometry (HRMS) [6,9,15-17]. Furthermore, repeated measurements were found to improve the adherence [18].

Amongst the analyzed body fluids, urine has become the matrix of choice for qualitative screening of antihypertensive drugs [9,17,19], but blood plasma [20] and oral fluid [15] were described to also be suitable. Almost all drugs or their metabolites can be detected in urine, and their complete absence guarantees that the medication has not been taken for a duration equivalent to several half-lives [6]. Urine can be obtained noninvasively in large volumes, and the drugs, as well as their metabolites, are usually concentrated [21]. Nevertheless, some major disadvantages of urine screening approaches were described, such as the elaborate sample collection, simple adulteration, and misclassification of drug adherence [22,23]. Compounds with a short half-life and/or low assay sensitivity could yield false negative classifications, while compounds with a long half-life and/or high assay sensitivity could yield false positive classifications for adherence [22]. Thus, recent data indicated that a thorough analysis of the blood serum or plasma may be beneficial over urine [22,24-27]. Accompanying the blood pressure measurement creates the opportunity to link the drug concentration to the blood pressure profile [22].

The present pilot study aimed to further elucidate the power of measuring blood plasma drug concentrations for adherence monitoring. Therefore, several objectives were pursued within the framework of the current study. First, to develop and validate an adherence monitoring method based on plasma quantification by ultra-high-performance (UHP) LC-ion trap mass spectrometry (ITMS). The method should cover frequently prescribed antihypertensive drugs $[28,29]$ and be developed and validated in accordance with guidelines of the European Medicines Agency (EMA) [30]. Second, to apply this method to assess adherence by using dose-dependent reference plasma concentration ranges. As the findings should finally be compared with those of an established, metabolite-based urine screening procedure by LC-HRMS [17], only plasma samples with matched urine samples that tested positive for antihypertensive drugs should be included in the applicability testing to enable a comparison of the adherence.

\section{Results}

\subsection{Development of the Analytical Procedure}

Nine antihypertensive drugs with different chemical properties were chosen for the current pilot study, including the beta-blockers bisoprolol, carvedilol, and metoprolol; the diuretics canrenone and torasemide; the ARBs candesartan, olmesartan, and valsar- 
tan; and the calcium antagonist amlodipine. A representative and reconstructed ion chromatogram of the $\mathrm{m} / \mathrm{z}$ of the antihypertensive drugs $(1 \mathrm{mg} / \mathrm{L}$, each) and the internal standard (IS) diazepam-d5 $(0.1 \mathrm{mg} / \mathrm{L})$ is depicted in Figure 1A. Different signal intensities despite the same concentration were observed, most probably due to differences in the ionization efficiency.
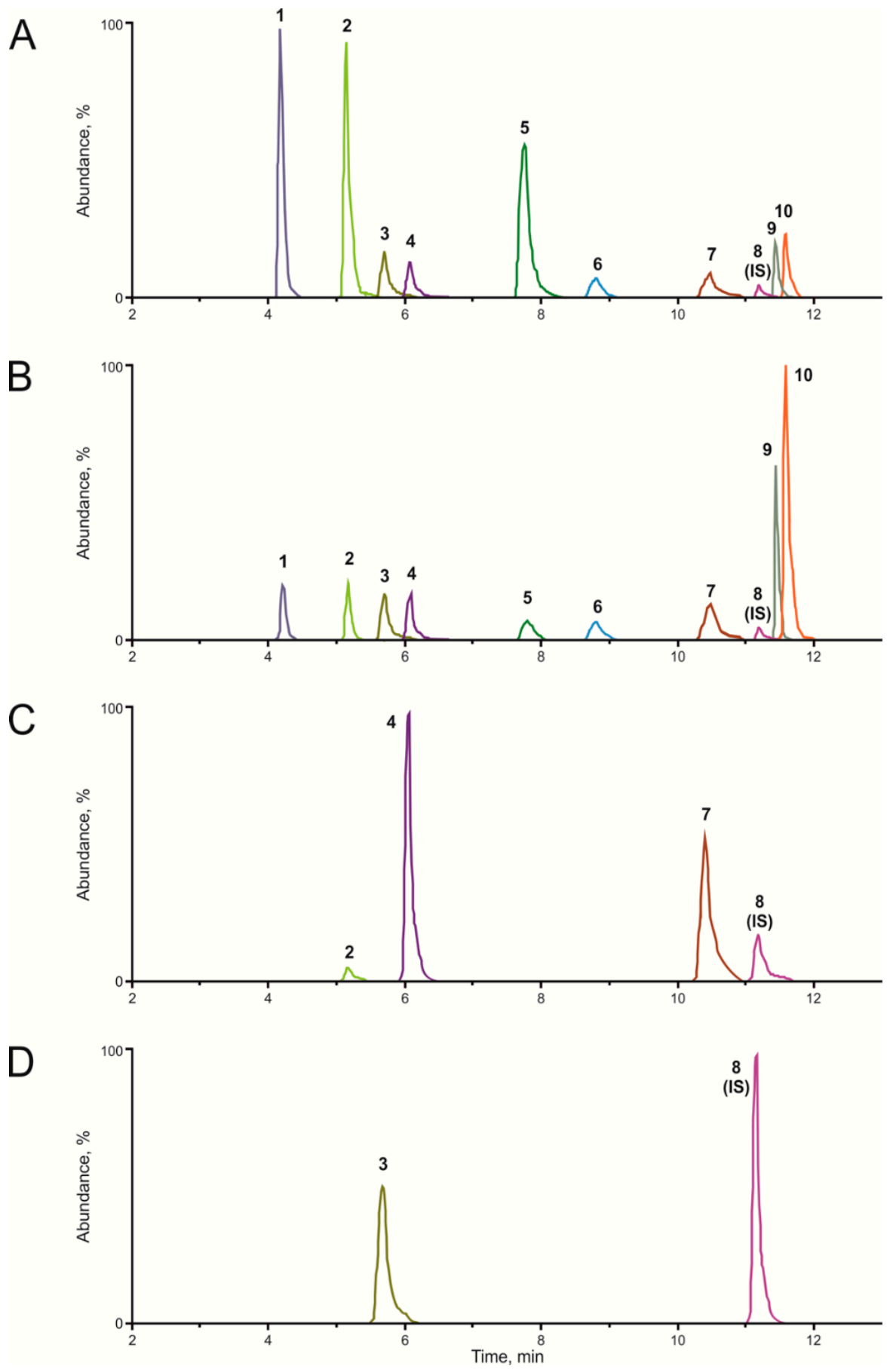

Figure 1. Reconstructed ion chromatograms of the $\mathrm{m} / \mathrm{z}$ of the antihypertensive drugs and the internal standard (IS) diazepam-d5 (1 metoprolol, 2 bisoprolol, 3 olmesartan, 4 torasemide, 5 carvedilol, 6 amlodipine, 7 candesartan, 8 IS diazepam-d5, 9 valsartan, and 10 canrenone). (A) Methanolic solution, antihypertensive drugs $1 \mathrm{mg} / \mathrm{L}$ each, IS $0.1 \mathrm{mg} / \mathrm{L}$. (B) Extracted high-quality control sample. (C) Extracted plasma sample after intake of bisoprolol, torasemide, and candesartan. (D) Extracted plasma sample after intake of olmesartan. 
Liquid-liquid extraction (LLE) was found as suitable sample preparation procedure. However, the addition of formic acid was crucial for the extraction of the acidic antihypertensive drugs candesartan, olmesartan, and valsartan from blood plasma. Repeated vortexing and shaking was needed to reliably homogenize the samples during sample preparation. Figure 1B shows a plasma extract at a high level of quality control (QC) (for concentrations, see Table 1) with an IS concentration of $10 \mathrm{ng} / \mathrm{mL}$. The sample preparation procedure was tested for interferences preventing the reducibility by determination of the coefficients of variation (CVs) of the peak area ratios after three extractions. CVs were found to be between $2 \%$ (torsemide) and $12 \%$ (metoprolol) and, thus, acceptable. All analytes were successfully separated via UHPLC within $12 \mathrm{~min}$, obtaining retention times $\left(t_{R}\right)$ between 4.2 and $11.7 \mathrm{~min}$ (details are given in Table 2). The total run time was $16 \mathrm{~min}$ per run. Furthermore, the smartMRM mode increased the UHPLC-ITMS sensitivity compared to autoMS ${ }^{n}$ mode, which is used by default for plasma screening and quantification aiming to detect intoxications in emergency toxicology [31].

Table 1. Final plasma concentrations $(\mathrm{ng} / \mathrm{mL})$ of the antihypertensive drugs in the six calibrator samples (Cal 1-6) and quality control samples (QC) at four levels (LLOQ, low, medium, and high), as well as weighting factors used in a linear calibration model. Lower limit of quantification (LLOQ), upper limit of quantification (ULOQ), and medium (Med).

\begin{tabular}{|c|c|c|c|c|c|c|c|c|c|c|c|}
\hline Analyte & $\begin{array}{c}\text { Cal 1 } \\
(\equiv \text { LLOQ) }\end{array}$ & Cal 2 & Cal 3 & Cal 4 & Cal 5 & $\begin{array}{c}\text { Cal } 6 \\
(=\mathrm{ULOQ})\end{array}$ & $\begin{array}{c}\mathrm{LLOQ} \\
\mathrm{QC}\end{array}$ & $\begin{array}{c}\text { Low } \\
\text { QC }\end{array}$ & $\begin{array}{c}\text { Med } \\
\text { QC }\end{array}$ & $\underset{\text { QC }}{\text { High }}$ & Weigh-ting \\
\hline Amlodipine & 10 & 50 & 100 & 150 & 200 & 250 & 10 & 30 & 120 & 240 & $1 / x$ \\
\hline Bisoprolol & 5 & 25 & 50 & 75 & 100 & 125 & 5 & 15 & 60 & 120 & $1 / x^{2}$ \\
\hline Candesartan & 10 & 50 & 100 & 150 & 200 & 250 & 10 & 30 & 120 & 240 & $1 / x^{2}$ \\
\hline Canrenone & 20 & 100 & 200 & 300 & 400 & 500 & 20 & 60 & 240 & 480 & $1 / x^{2}$ \\
\hline Carvedilol & 1 & 5 & 10 & 15 & 20 & 25 & 1 & 3 & 12 & 24 & $1 / x^{2}$ \\
\hline Metoprolol & 10 & 50 & 100 & 150 & 200 & 250 & 10 & 30 & 120 & 240 & $1 / x^{2}$ \\
\hline Olmesartan & 5 & 25 & 50 & 75 & 100 & 125 & 5 & 15 & 60 & 120 & $1 / x$ \\
\hline Torasemide & 5 & 25 & 50 & 75 & 100 & 125 & 5 & 15 & 60 & 120 & equal \\
\hline Valsartan & 10 & 50 & 100 & 150 & 200 & 250 & 10 & 30 & 120 & 240 & $1 / x$ \\
\hline
\end{tabular}

Table 2. Precursor ion $m / z$ after positive ionization $\left(\mathrm{M}+\mathrm{H}^{+}\right)$, retention order, and retention times $\left(\mathrm{t}_{\mathrm{R}}\right)$ of the antihypertensive drugs and internal standards (IS).

\begin{tabular}{cccc}
\hline Analyte & Precursor Ion $\mathbf{m / z}$ & Retention Order & $\mathbf{t}_{\mathbf{R}}, \mathbf{m i n}$ \\
\hline Amlodipine & 409.15 & 6 & 8.8 \\
Bisoprolol & 326.23 & 2 & 5.2 \\
Candesartan & 441.17 & 7 & 10.5 \\
Canrenone & 341.21 & 10 & 11.7 \\
Carvedilol & 407.20 & 5 & 7.8 \\
Diazepam-d5 (IS) & 290.11 & 8 & 11.3 \\
Metoprolol & 268.19 & 1 & 4.2 \\
Olmesartan & 447.21 & 3 & 5.7 \\
Torasemide & 349.13 & 4 & 6.1 \\
Valsartan & 436.23 & 9 & 11.5 \\
\hline
\end{tabular}

\subsection{Method Validation}

Atorvastatin-d5 and atorvastatin, as well as diazepam-d5 and diazepam, were found to coelute and were therefore tested for the effects of the nondeuterated compounds on the peak area of the deuterated compounds. Ion suppression of atorvastation-d5 $(-17 \%)$ was detected in the presence of atorvastatin, while diazepam did not influence the diazepam-d5 peak area. Therefore, further analyses were performed using only diazepam-d5 as IS.

Method validation was performed in accordance with the "Guideline on bioanalytical method validation" of the EMA [30]. Selectivity was given for all antihypertensive drugs, as well as for the IS diazepam-d5. No carryover was observed, except for torasemide with an analyte response greater than $20 \%$ of its lower limit of quantification (LLOQ), which corresponded to the concentration of Cal 1. However, the analyte response was not greater 
than $20 \%$ of Cal 2. After five washing runs injecting an eluent mixture A/B (1:1, v/v, see Materials and Methods) immediately after the analysis of $\mathrm{Cal} 6$, the analyte response was below $20 \%$ of the LLOQ of torasemide.

A linear calibration model was successfully applied using a six-point calibration of all analytes. However, different weighing factors (equal, $1 / x, 1 / x^{2}$ ) had to be used. The details are given in Table 1 . The acceptance criteria (AC) were checked for each individual calibration. Calibration parameters such as slope, y-axis intercept, and the deviation of the back-calculated concentrations of the three calibrations acquired during the validation procedure are available in the Supplementary Materials (Table S1). If the AC for the back-calculated concentrations were not fulfilled, this calibration standard sample was rejected for the specific analyte, and the calibration without this calibration standard was reevaluated, including a regression analysis according to the guidelines [30]. Nevertheless, at least five out of six calibration standards fulfilled these criteria for each calibration, and neither Cal 1 nor Cal 6 had to be excluded in accordance with the EMA guidelines.

Concerning the matrix effect experiments, Table 3 summarizes the IS-normalized matrix factors (MF) of all analytes, ranging from 0.82 (candesartan) to 2.56 (amlodipine) for the low QC and 0.96 (candesartan and olmesartan) to 1.37 (amlodipine) for the high QC. CVs were not greater than $15 \%$ for bisoprolol, canrenone, and torasemide in the case of low QC, while all other analytes exceeded the AC with CVs between 17\% (metoprolol) and 42\% (valsartan). For high QC, CVs were not greater than 15\% for all analytes except amlodipine $(16 \%)$, candesartan, olmesartan (both $17 \%)$, and valsartan $(25 \%)$. In general, the matrix effects were observed to be more pronounced in the case of low analyte concentrations, but the difference between the IS-normalized MF determined for low and high QC was highest in the case of amlodipine.

Table 3. Internal standard (IS)-normalized matrix factors of the analytes and coefficients of variation (CV) derived from measurements of six lots of the matrix at a low (low QC, see Table 1) and a high concentration (high QC, see Table 1).

\begin{tabular}{|c|c|c|c|c|}
\hline \multirow{2}{*}{ Analyte } & Low QC & \multirow{2}{*}{$\mathrm{CV}, \%$} & High QC & \multirow{2}{*}{$\mathrm{CV}, \%$} \\
\hline & IS-Normalized Matrix Factor & & IS-Normalized Matrix Factor & \\
\hline Amlodipine & 2.54 & $26^{*}$ & 1.37 & $16^{*}$ \\
\hline Bisoprolol & 1.12 & 11 & 1.07 & 13 \\
\hline Candesartan & 0.82 & 24 * & 0.96 & $17^{*}$ \\
\hline Canrenone & 1.10 & 11 & 0.99 & 10 \\
\hline Carvedilol & 1.15 & $19 *$ & 1.08 & 7 \\
\hline Metoprolol & 1.16 & $17 *$ & 1.07 & 10 \\
\hline Olmesartan & 0.95 & $22 *$ & 0.96 & $17 *$ \\
\hline Torasemide & 1.06 & 12 & 1.08 & 12 \\
\hline Valsartan & 1.12 & $42 *$ & 1.07 & $25 *$ \\
\hline
\end{tabular}

Values outside the recommended European Medicines Agency (EMA) guideline limits [30] are marked with an asterisk $\left.{ }^{*}\right)$.

The accuracy of an analytical method describes the closeness of the determined value obtained by the method to the nominal concentration of the analyte. The precision of the analytical method describes the closeness of repeated individual measures of the analyte [30]. The within-run accuracy was found to be acceptable with mean concentrations within $\pm 15 \%$ of the nominal values for the low, medium, and high QC samples (1-15\%) and within $\pm 20 \%$ for the LLOQ QC samples (2-15\%) for all analytes, except for amlodipine (medium QC, 17\%), carvedilol (medium QC, 21\%), torasemide (LLOQ QC, 34\% and low QC, 20\%), and valsartan (low QC, 20\%). The between-run accuracy was found to be acceptable, with mean concentrations within $\pm 15 \%$ of the nominal values for the low, medium, and high QC samples (0-15\%) and within $\pm 20 \%$ for the LLOQ QC samples (1-19\%) for all analytes, except for carvedilol (LLOQ QC, 28\%), olmesartan (LLOQ QC, $26 \%$ ), and torasemide (low QC, 26\%). The within-run and between-run precision were expressed as the CV and found to be acceptable with CV values within $15 \%$ for the low, medium, and high QC samples (1-14\%) and within 20\% for the LLOQ QC samples (3-20\%) for all analytes with the exception of valsartan (within-run precision, medium QC, CV 19\%). 
After the dilution of samples spiked above the ULOQ (= Cal 6), the determined concentrations were found to be precise (CVs of 6-15\%) for all analytes. The determined values were also found to be accurate for bisoprolol, candesartan, canrenone, olmesartan, and valsartan (1-12\% variation). In the case of amlodipine (25\%), carvedilol, metoprolol (both $30 \%$ ), and torasemide (17\%), the AC of the EMA concerning accuracy after dilution were exceeded.

Methanolic stock solutions were found to be stable at $-20{ }^{\circ} \mathrm{C}$ with $\mathrm{CVs}$ below $15 \%$ for all analytes. All analytes were found to be stable in plasma extracts after $20 \mathrm{~h}$ in the autosampler at $8{ }^{\circ} \mathrm{C}$, except for amlodipine with a decrease of $16 \%$ compared to the nominal concentration.

\subsection{Applicability and Assessment of Adherence by Using the Dose-Dependent Reference Plasma Concentration Range}

Applicability testing was performed using 19 different plasma samples. A total of 28 antihypertensive drugs were prescribed $(7 \times$ bisoprolol, $5 \times$ candesartan, $1 \times$ canrenone, $1 \times$ carvedilol, $2 \times$ metoprolol, $3 \times$ olmesartan, $6 \times$ torasemide, and $3 \times$ valsartan), and all of them could successfully be identified in plasma. Three different antihypertensive drugs were present in two samples (ID 6 and 14) and two drugs in five samples (IDs 4, $11,12,13$, and 18), while one drug was quantified in the remaining 12 plasma samples (IDs 1, 2, 3, 5, 7, 8, 9, 10, 15, 16, 17, and 19). Reconstructed ion chromatograms after the analysis of two samples are given in Figure 1C (sample ID 14) and Figure 1D (sample ID 10). Previously, matching urine samples were tested positive for the antihypertensive drugs and/or their metabolites using an established, metabolite-based urine screening procedure by LC-HRMS [17].

The plasma quantification results given in Table 4 also contained the therapeutic plasma concentration range described in the literature [32]; they prescribed daily doses of the antihypertensive drugs and calculated dose-dependent reference plasma concentration ranges with the underlaying pharmacokinetic parameters taken from Baselt [33]. The calculated dose-dependent reference plasma concentration ranges were defined to comprise $\mathrm{C}_{\min -20 \%}$ to $\mathrm{c}_{\max }$. The trough plasma concentration $24 \mathrm{~h}$ after the latest drug intake, $\mathrm{c}_{\min }$ was calculated using the elimination half-life $t_{1 / 2}$, peak plasma concentration $c_{\max }$, and the corresponding time after intake when $c_{\max }$ was reached $\left(t_{\max }\right)$ for the corresponding dose described by Baselt [33]. The shortest given elimination half-life $t_{1 / 2}$ was always used for the calculation. A subtraction of $20 \%$ from $c_{\min }$ led to $c_{\min }-20 \%$, which was defined as lower end of the calculated dose-dependent reference plasma concentration range. The peak plasma concentration $c_{\max }$ was defined as the upper end. A summary of the pharmacokinetic parameters used for calculation of the reference plasma concentration ranges, as well as an example for such a calculation, are available as Supplementary Materials Tables S2 and S3, respectively.

The dose used for the calculation of the reference plasma concentration range was given in brackets if it was different from the patient's prescribed daily dose. The measured plasma concentration was above the ULOQ of olmesartan, torasemide, or valsartan, respectively, in the case of six samples (IDs 3, 6, 8, 11, 14, and 19), which were diluted (1:10 with pooled blank plasma), extracted, and reanalyzed. The measured plasma concentration was still above the ULOQ of valsartan in the case of one sample (IDs 11), and it was thus diluted (1:20 with pooled blank plasma), extracted, and reanalyzed.

Compared to the therapeutic plasma concentration ranges described in the literature [32], the determined plasma concentrations of 12 out of 28 antihypertensive drugs were within (43\%), 15 below (53\%), and one above (4\%) the given ranges. Compared to the calculated dose-dependent reference plasma concentration ranges, the determined plasma concentrations of 21 out of 28 antihypertensive drugs were within ( $75 \%$, three below (11\%), and two above $(7 \%)$ the range. In the case of two antihypertensive drugs, the determined plasma concentrations were below the LLOQ $(7 \%)$. However, the latter was higher than $\mathrm{C}_{\min -20 \%}$ in both cases. 
Table 4. Quantification results of 19 plasma samples containing 28 antihypertensive drugs in total, along with the prescribed daily doses, therapeutic plasma concentration ranges according to Schulz et al. [32], elimination half-lives $t_{1 / 2}$, time after intake when the peak plasma concentration was reached $\left(t_{\max }\right)$, and calculated dose-dependent reference plasma concentration ranges $\left(c_{\min -20 \%-c_{\max }}\right)$. The dose used for calculation of the reference plasma concentration range was given in parentheses if it was different from the patient's prescribed daily dose. Plasma concentrations below the LLOQ are indicated by < LLOQ. Plasma concentrations below the given concentration ranges are marked by $(\downarrow)$, within by $(\leftrightarrow)$, and above by $(\uparrow)$. If the measured plasma concentration cannot be classified using the given concentration ranges, they are marked by (?).

\begin{tabular}{|c|c|c|c|c|c|c|c|}
\hline Analyte & Sample ID & $\begin{array}{c}\text { Measured Plasma } \\
\text { Conc. ng/mL }\end{array}$ & $\begin{array}{c}\text { Daily Dose, } \\
\text { mg }\end{array}$ & $\begin{array}{c}\text { Therapeutic Plasma } \\
\text { Conc., ng/mL [32] }\end{array}$ & $\begin{array}{c}\mathbf{t}_{1 / 2}, \mathbf{h} \\
{[33]}\end{array}$ & $\mathbf{t}_{\text {max }}, \mathbf{h}$ & $\begin{array}{l}\text { Calculated Reference } \\
\text { Plasma Conc., ng/mL }\end{array}$ \\
\hline Bisoprolol & $\begin{array}{c}2 \\
4 \\
6 \\
9 \\
12 \\
13 \\
14\end{array}$ & $\begin{array}{c}5.9 \\
27 \\
17 \\
9 \\
17 \\
<\operatorname{LLOQ}(5 \mathrm{ng} / \mathrm{mL}) \\
5.5\end{array}$ & $\begin{array}{c}2.5 \\
10 \\
5 \\
5 \\
5 \\
2.5 \\
5\end{array}$ & $\begin{array}{c}10-100(\downarrow) \\
10-100(\leftrightarrow) \\
10-100(\leftrightarrow) \\
10-100(\downarrow) \\
10-100(\leftrightarrow) \\
10-100(\downarrow) \\
10-100(\downarrow)\end{array}$ & $7-15$ & 2 & $\begin{array}{c}2.0-21(5 \mathrm{mg})(\leftrightarrow) \\
4.8-52(\leftrightarrow) \\
2.0-21(\leftrightarrow) \\
2.0-21(\leftrightarrow) \\
2.0-21(\leftrightarrow) \\
2.0-21(5 \mathrm{mg})(?) \\
2.0-21(\leftrightarrow)\end{array}$ \\
\hline Candesartan & $\begin{array}{l}12 \\
14 \\
15 \\
16 \\
17\end{array}$ & $\begin{array}{c}66 \\
160 \\
92 \\
83 \\
49\end{array}$ & $\begin{array}{c}8 \\
32 \\
32 \\
16 \\
16\end{array}$ & $\begin{array}{c}80-400(\downarrow) \\
80-400(\leftrightarrow) \\
80-400(\leftrightarrow) \\
80-400(\leftrightarrow) \\
80-400(\downarrow)\end{array}$ & $8-13$ & 4 & $\begin{array}{c}9.2-61(\uparrow) \\
39-260(\leftrightarrow) \\
39-260(\leftrightarrow) \\
18-120(\leftrightarrow) \\
18-120(\leftrightarrow)\end{array}$ \\
\hline Canrenone & 4 & 90 & 50 & $50-500(\leftrightarrow)$ & $13-23$ & 2 & $160-600(100 \mathrm{mg})(\downarrow)$ \\
\hline Carvedilol & 18 & 2.4 & 6.25 & $20-300(\downarrow)$ & $4-7$ & 1 & $0.36-58(12.5 \mathrm{mg})(\leftrightarrow)$ \\
\hline Metoprolol & $\begin{array}{l}5 \\
7 \\
\end{array}$ & $\begin{array}{c}38 \\
<\operatorname{LLOQ}(10 \mathrm{ng} / \mathrm{mL})\end{array}$ & $\begin{array}{c}50 \\
100\end{array}$ & $\begin{array}{c}20-600(\leftrightarrow) \\
20-600(\downarrow)\end{array}$ & $2.5-7.5$ & $\begin{array}{l}1 \\
2\end{array}$ & $\begin{array}{l}0.10-72(\leftrightarrow) \\
0.26-140(?)\end{array}$ \\
\hline Olmesartan & $\begin{array}{c}1 \\
3 \\
10\end{array}$ & $\begin{array}{c}52 \\
1100 \\
14\end{array}$ & $\begin{array}{l}40 \\
40 \\
20\end{array}$ & $\begin{array}{l}100-1000(\downarrow) \\
100-1000(\uparrow) \\
100-1000(\downarrow)\end{array}$ & $6-15$ & 2 & $\begin{array}{l}55-830(\downarrow) \\
55-830(\uparrow) \\
26-390(\downarrow)\end{array}$ \\
\hline Torasemide & $\begin{array}{c}6 \\
8 \\
11 \\
13 \\
14 \\
18\end{array}$ & $\begin{array}{c}41 \\
240 \\
18 \\
30 \\
170 \\
9.2 \\
\end{array}$ & $\begin{array}{c}10 \\
10 \\
10 \\
10 \\
5 \\
2.5\end{array}$ & $\begin{array}{c}64-2000(\downarrow) \\
64-2000(\stackrel{\leftrightarrow}{)}) \\
64-2000(\downarrow) \\
64-2000(\downarrow) \\
64-2000(\leftrightarrow) \\
64-2000(\downarrow)\end{array}$ & $2-6$ & 1 & $\begin{array}{c}0.47-1600(\leftrightarrow) \\
0.47-1600(\leftrightarrow) \\
0.47-1600(\leftrightarrow) \\
0.47-1600(\leftrightarrow) \\
0.47-1600(10 \mathrm{mg})(\leftrightarrow) \\
0.47-1600(10 \mathrm{mg})(\leftrightarrow)\end{array}$ \\
\hline Valsartan & $\begin{array}{c}6 \\
11 \\
19\end{array}$ & $\begin{array}{c}920 \\
3000 \\
410\end{array}$ & $\begin{array}{l}320 \\
320 \\
320\end{array}$ & $\begin{array}{c}800-6000(\leftrightarrow) \\
800-6000(\leftrightarrow) \\
800-6000(\downarrow)\end{array}$ & $4-12$ & 3 & $\begin{array}{l}240-11,000(\leftrightarrow) \\
240-11,000(\leftrightarrow) \\
240-11,000(\leftrightarrow)\end{array}$ \\
\hline
\end{tabular}

\section{Discussion}

\subsection{Development and Validation of the Analytical Procedure}

The chosen antihypertensive drugs, including beta-blockers, ARBs, diuretics, and a calcium antagonist, are frequently prescribed [28]. Canrenone can be used as diuretic drug itself but also represents the active main metabolite of its prodrug spironolactone [34]. The method is therefore suitable for the adherence monitoring of both drugs. All of these antihypertensive drugs are also available in fixed-dose combinations, the use of which is recommended by the current guidelines [28].

Only $100 \mu \mathrm{L}$ of blood plasma were required for the analysis. The duration of a single analytical run (including equilibration) of $16 \mathrm{~min}$ is expected to be appropriate and applicable in the daily laboratory routine. A single injection and analysis per sample saved run time and was found to be adequate during the method development. Diazepam-d5 was chosen as a suitable IS. In contrast to atorvastatin-d5, which provided a higher $t_{R}(12.4 \mathrm{~min})$ than all the antihypertensive drugs, diazepam-d5 eluted between candesartan and valsartan (see Table 2). Furthermore, neither ion suppression nor the enhancement of its peak area was detected in the presence of coeluting diazepam. Remane et al. [35] demonstrated the importance of ion suppression and enhancement experiments for developing LCbased mass spectrometry procedures if the baseline separation is not possible, and the current results confirmed their findings. Some patients may be advised to take atorvastatin, diazepam, and antihypertensive drugs at the same time. Ion suppression and enhancement of the peak area of the IS may lead to false quantification results. Atorvastatin suppressed 
the atorvastatin-d5 peak area, which may lead to overestimation of the antihypertensive drug plasma concentrations. In contrast, diazepam did not influence the diazepam-d5 area, and the current method is therefore also applicable for patients advised to take diazepam. No other analytes were tested for ion suppression or enhancement due to sufficient chromatographic separation (see Figure 1A).

The bioanalytical methods applied for measuring drug concentrations in biological matrices have to be validated in order to provide reliable results [30]. The developed method was fully validated according to the EMA guidelines [30]. The limitations observed during validation will be discussed in the following. Due to the carryover of torasemide, several washing runs injecting the eluent mixture A/B ( $1: 1, v / v$, see Materials and Methods) are recommended after the analysis of $\mathrm{Cal} 6$, high $\mathrm{QC}$, or torasemide-containing patient samples to ensure that the analyte response is below $20 \%$ of the LLOQ. Based on the validation procedure, five washing runs were recommended. However, this option is quite time-consuming $(>1 \mathrm{~h})$. Washing runs are also recommended if patient samples containing plasma concentrations above those of Cal 6 were analyzed. In these cases, the washing run should be checked for carryover of the analyte. In the case of torasemide, the elevation of its LLOQ to the concentration of Cal $2(25 \mathrm{ng} / \mathrm{mL})$ represents a less time-consuming alternative, which may complicate the adherence assessment of low torasemide plasma concentrations. The first option, consisting of washing runs, was chosen for the subsequent validation and application experiments described in the current study, but the latter may be more suitable for routine application of the presented method.

The EMA guidelines do not define AC for the MF but for the CV of the IS-normalized MF calculated from six lots of matrix that should not exceed $15 \%$. However, CVs were $>15 \%$ in the case of six analytes for low QC and in the case of four analytes for high QC. However, half of them (two analytes for low QC and three analytes for high QC) had CVs between $15 \%$ and $20 \%$. Other guidelines for the quantitative determination of drugs, such as the "Recommendations of criteria for development and validation of analytical methods for estimating concentrations of drugs in blood to be used in 24/7 clinical toxicology" published by the Society of Toxicological and Forensic Chemistry (GTFCh) [36], include different instructions for assessing matrix effects. The GTFCh recommendations stated that the matrix effect should not exceed $\pm 30 \%$. This means, for the current study, that IS-normalized MF between 0.70 and 1.30 would be acceptable. According to this recommendation, all analytes fulfilled the AC except for amlodipine in low and high QC.

According to the EMA guidelines, the mean concentration determined in the accuracy experiments should be within $\pm 15 \%$ of the nominal value (LLOQ $\pm 20 \%$ ) [30]. The accuracy of the AC was not fulfilled for the individual concentration levels in the case of five antihypertensive drugs (amlodipine, carvedilol, olmesartan, torasemide, and valsartan). However, the AC were still within $25 \%$ in most cases. For precision experiments, CVs should not exceed $15 \%$ (LLOQ 20\%), and the precision AC were only exceeded in the case of valsartan but still within $20 \%$. In comparison, the aforementioned recommendations of the GTFCh allow $30 \%$ of the nominal value of two QC levels at the lower $(20 \%)$ and upper $(80 \%)$ end of the calibration range, as well as the precision of $<30 \%$ [36]. Accordingly, the current validation experiments would be in line with those recommendations, except for torasemide concerning the within-run accuracy at the LLOQ QC level (34\%) supporting the elevation of the LLOQ of torasemide to the concentration of Cal 2.

After dilution, the determined concentrations turned out to be precisely in accordance with the EMA AC, while the quantification results of four antihypertensive drugs exceeded the accuracy AC of the EMA. Nevertheless, the quantified concentrations did not exceed the cut-off of $\pm 30 \%$ of the nominal value, which was recommended by the GTFCh.

According to the EMA guidelines, evaluation of the stability must be carried out to ensure that every step taken during the sample preparation and sample analysis, as well as the storage conditions used, do not affect the concentration of the analyte [30]. The current experiments investigated whether the storage conditions used for the spiking 
solutions, as well as the conditions in the autosampler during short-term storage of the plasma extracts, influenced the analytes' concentrations. The stability of methanolic stock solutions could be ensured for the whole validation process and subsequent applicability testing. However, it should be regularly retested in case the current method is used for routine work. Extracted plasma samples were stable for at least $20 \mathrm{~h}$ in the autosampler. It is therefore recommended to finalize the batch analysis within this timeframe.

The stability of the analytes in the current sample matrix plasma was not investigated, as this information was available from the literature [33]. Baselt described all analytes to be stable for $24 \mathrm{~h}$ in plasma if stored at room temperature and for at least a month if stored in the freezer. This means that plasma should be separated by centrifugation immediately after sample collection. If samples cannot be transferred to the laboratory within $24 \mathrm{~h}$, it is recommended to freeze them and ship them frozen to ensure the analytes' stability.

In summary, the current method was successfully validated and fulfilled the AC of the EMA and/or the GTFCh $[30,36]$. It is therefore regarded as applicable for adherence monitoring of hypertensive patients by drug quantification in blood plasma. The only exception is amlodipine, whose IS-normalized MF exceeded the AC of both guidelines in low and high QC. Furthermore, amlodipine is known to be photosensitive [26], but all amlodipine-containing solutions were handled in amber-colored vials during method development and validation. The amlodipine plasma concentrations should be interpreted with caution, and clinicians must be advised to immediately protect amlodipine-containing plasma samples from light. As both high matrix effects and photosensitivity might impact a reliable quantification, amlodipine was not included in the applicability study.

\subsection{Applicability and Assessment of Adherence by Using the Dose-Dependent Reference Plasma Concentration Range}

Applicability was tested using 19 plasma samples containing 28 antihypertensive drugs. Each of the eight antihypertensive drugs that passed the validation procedure contained at least in one plasma sample (see Table 4). Interpretation of the analytical results is one crucial step. Classification of the plasma concentrations is more sophisticated and time-consuming than the evaluation of qualitative urine screening results, where the presence or absence of a drug and/or its metabolites represents the main criterion.

Thus, the measured plasma concentrations were compared to the plasma concentrations described as therapeutic ("normal") in the paper by Schulz et al. [32]. These data are intended to help toxicologists to decide whether a patient may suffer from an intoxication and do not focus on adherence monitoring. Only a single range is given for each drug, independent of the prescribed daily dose. Plasma concentrations of only 12 antihypertensive drugs were found to be within the described therapeutic range, while even more were below it. These findings are in accordance with the observations by Ritscher et al. [25]. They developed a plasma quantification procedure including four beta-blockers (amongst them, bisoprolol and metoprolol) and four diuretics (amongst them, canrenone and torasemide) with a focus on the adherence assessment and compared their quantification results with the therapeutic plasma concentration ranges according to the article by Schulz et al. from 2012 [37]. About 30\% were below the described therapeutic concentration ranges, and the authors concluded that these ranges cannot be used to evaluate adherence, as drug ingestion was monitored in their study. Therefore, Ritscher et al. used a dose-related concentration approach and compared measured concentrations with trough serum drug concentrations calculated individually for each patient using data from pharmacokinetic studies, including bioavailability, total body clearance, and elimination rate constants [25]. Rognstad et al. also addressed the problem of lacking serum reference ranges of antihypertensive drugs and preventing the widespread use of therapeutic drug monitoring in cardiology [38]. Nevertheless, the serum concentrations measured by Ritscher et al. [25] were comparable to the plasma concentrations determined in the current study. In the case of bisoprolol, Ritscher et al. described the trough serum levels between 8.9 and $41 \mathrm{ng} / \mathrm{mL}$ for patients with prescribed daily doses between $2.5 \mathrm{and} 10 \mathrm{mg}$. Two trough serum concentrations each were described for canrenone $(26$ and $48 \mathrm{ng} / \mathrm{mL}$, daily spironolactone 
dose $25 \mathrm{mg}$, each), as well as metoprolol (6.6 and $13 \mathrm{ng} / \mathrm{mL}$, daily dose 50 or $100 \mathrm{mg}$, respectively). Concerning torasemide, Ritscher et al. reported trough serum levels between 18 and $370 \mathrm{ng} / \mathrm{mL}$ for patients with prescribed daily doses of 5 or $10 \mathrm{mg}$ [25].

In the current study, drug intake was not monitored, and the exact time between intake and sampling was unknown, as is usually the case for outpatients. Therefore, adherence cannot be assumed, even if matching urine samples were tested positive for the antihypertensive drugs and/or their metabolites [17]. Instead, a simplified assessment approach was used by calculating the dose-dependent reference plasma concentration range $\left(\mathrm{c}_{\min }-20 \%-\right.$ $c_{\max }$ ) for each antihypertensive drug by using data from Baselt [33], such as the peak plasma concentrations $c_{\max }$, the time at which $c_{\max }$ was reached $\left(t_{\max }\right)$, and the elimination half-life $t_{1 / 2}$. The latter usually represents a range, and the shortest given half-life was used for the calculations. The dosing regimens were simplified by calculating the total daily dose with a hypothetical dosing interval of $24 \mathrm{~h}$ according to Hiemke et al. [39]. The leastexpected plasma concentration at $24 \mathrm{~h}$ after the latest drug intake (trough concentration) was $\mathrm{c}_{\min }$. A subtraction of $20 \%$ led to $\mathrm{c}_{\min -20 \%}$ which was defined as the lower end of the reference plasma concentration range to exclude the influence of interindividual differences and measurement uncertainties. As Baselt [33] did not report peak plasma concentrations for all doses prescribed to the patients, the quantification results were evaluated using the most comparable dose of which the peak plasma concentration $c_{\max }$ was available.

The determined plasma concentrations of 21 out of 28 antihypertensive drugs were found to be within the calculated dose-dependent reference plasma concentration ranges underlining the importance of including the individual daily dose and trough concentrations. Three plasma concentrations were below the calculated reference ranges, but the drugs could successfully be identified in plasma. In the case of canrenone (sample ID 4), the patient was advised to take only $50 \mathrm{mg}$ per day, but the peak plasma concentration $c_{\max }$ was only available after an intake of $100 \mathrm{mg}$. In the case of olmesartan (sample ID 1 and 10), the concentrations were slightly below the lower limit of the reference plasma concentration ranges. In all three cases, a follow-up analysis may be recommended.

Two plasma concentrations were above the calculated reference concentration ranges. However, the upper limit of the reference range was defined by the peak plasma concentration $c_{\max }$. This value only reflects the mean value of a patient collective. In conclusion, higher peak plasma concentrations may be expected in some patients, leading to the conclusion that patients with plasma concentrations exceeding the given peak plasma concentration $c_{\max }$ (e.g., sample ID 3 and 12) are considered as adherent. Furthermore, the concentrations of olmesartan, torasemide, or valsartan were above the ULOQ in the case of six samples, which were subsequently diluted to determine the plasma concentration. It should be mentioned that this dilution step was not necessary to assess the adherence, as the ULOQ (125 ng/mL for olmesartan and torasemide and $250 \mathrm{ng} / \mathrm{mL}$ for valsartan) was within the calculated dose-dependent reference plasma concentration range in all cases.

In two cases containing bisoprolol (sample ID 13) or metoprolol (sample ID 7), the determined plasma concentrations were below the LLOQ, which was higher than $c_{\min -20 \%}$. Therefore, an adherence assessment was not possible in both cases. However, it must be mentioned that additional torasemide in the case of sample ID 13 was within the reference plasma concentration range. Furthermore, if the torasemide LLOQ would be elevated to the concentration of Cal $2(25 \mathrm{ng} / \mathrm{mL})$ due to the carryover, the adherence of the patients with sample IDs 11 and 18 to torasemide could not be assessed anymore.

Of course, the current procedure has limitations. First of all, calculating the dosedependent reference plasma concentration ranges is time-consuming, but as soon as they are available, they can be used for patients receiving the same daily dose. Furthermore, most data from Baselt described plasma concentrations after a single dose [33], and higher plasma concentrations may be expected in a steady state. Interindividual differences, for example, in the expression of metabolic enzymes were not considered. Finally, some analytes were only contained in a single plasma sample, as only a limited number of samples was analyzed. As a future perspective, more plasma samples should be analyzed 
to further evaluate and improve the current dose-dependent reference plasma concentration ranges approach.

In summary, the measured plasma concentrations of 12 out of 19 samples were within the calculated reference plasma concentration ranges for all analyzed antihypertensive drugs $(65 \%)$, of two times above $(10 \%)$, and of three times below $(15 \%)$. The adherence of sample IDs 7 and 13 could not be assessed, due to bisoprolol or metoprolol plasma concentrations and $\mathrm{c}_{\min -20 \%}$ below the LLOQ. Fourteen out of 19 samples were classified as adherent (75\%) and three as nonadherent (IDs 1, 4, and 10).

\section{Materials and Methods}

\subsection{Chemicals, Reagents, and Human Biosamples}

Amlodipine besylate was purchased from Pfizer (Karlsruhe, Germany); canrenone from the European Directorate for the Quality of Medicines \& HealthCare (Strasbourg, France); bisoprolol fumarate, metoprolol tartrate, carvedilol, candesartan, valsartan, olmesartan, torasemide, and a diazepam- $\mathrm{d} 5$ solution $(1 \mathrm{mg} / \mathrm{mL}$ in methanol) from LGC Standards (Wesel, Germany); atorvastatin-d5 calcium salt from Alsachim (Shimadzu Group, Illkirch Graffenstaden, France); and methanol, acetonitrile (ACN), diethyl ether, ethyl acetate, and natrium sulfate from VWR International GmbH (Darmstadt, Germany), as well as ammonium formate and formic acid from Merck KGaA (Darmstadt, Germany). All chemicals were of analytical grade. Water was purified with a Millipore filtration unit (18.2 $\Omega \times \mathrm{cm}$ water resistance) from Merck (Darmstadt, Germany). Drug-free pooled human blank plasma was obtained from a local blood bank. The analysis of human samples was approved by the local ethics committee to assess medication adherence using toxicological analyses objectively and complied with the Declaration of Helsinki. All patients provided written informed consent.

\subsection{Preparation of Stock Solutions for Calibrators and Quality Controls}

Stock solutions $(1 \mathrm{mg} / \mathrm{mL})$ of the antihypertensive drugs and of the IS atorvastatin-d5 were prepared in methanol. All solutions were stored at $-20^{\circ} \mathrm{C}$. Methanolic mix solutions for the calibrators and QC samples were prepared using different stock solutions and freshly spiked in pooled human blank plasma during sample preparation. Amlodipinecontaining solutions were handled in amber-colored vials during method development and validation. Final plasma concentrations of the calibrators (Cal 1-6), as well as QC samples (at four levels: LLOQ, low, medium, and high), are given in Table 1.

\subsection{Sample Preparation}

Plasma samples were prepared by LLE. First, $100 \mu \mathrm{L}$ of blood plasma, $10 \mu \mathrm{L}$ of formic acid $(100 \%), 10 \mu \mathrm{L}$ of diazepam-d5 (100 ng/mL in methanol), and $10 \mu \mathrm{L}$ of methanol were mixed and vortexed for $10 \mathrm{~s}$. For preparation of the calibrator and QC samples, methanol was replaced by $10 \mu \mathrm{L}$ of the respective mix solution (concentrations given in Table 1). Afterwards, $100 \mu \mathrm{L}$ of a saturated sodium sulfate solution were added, the mixture was vortexed for $10 \mathrm{~s}$, and $500 \mu \mathrm{L}$ of diethyl ether-ethyl acetate $(1: 1, v / v)$ were added. After another vortexing step (10 s), manual shaking for $30 \mathrm{~s}$, and centrifugation ( $5 \mathrm{~min}, 18,407 \times g$ ), $300 \mu \mathrm{L}$ of the clear organic phase were transferred to an amber-colored vial and evaporated to dryness at $30^{\circ} \mathrm{C}$ under a stream of nitrogen. The residue was reconstituted in $40 \mu \mathrm{L}$ of a mixture of eluents $\mathrm{A}$ and $\mathrm{B}(60: 40, v / v, 2-\mathrm{mM}$ aqueous ammonium formate containing $0.1 \%$ formic acid and $1 \% \mathrm{ACN}$ : ACN containing $2-\mathrm{mM}$ ammonium formate, $0.1 \%$ formic acid, and $1 \%$ water). Reproducibility of the sample preparation procedure was tested by determination of the CVs of the peak area ratios of the analyte $\left(\mathrm{m} / \mathrm{z}\right.$ of $\mathrm{M}^{+} \mathrm{H}^{+}$, given in Table 2) and the IS diazepam-d5 at a plasma concentration of $100 \mathrm{ng} / \mathrm{mL}$, each, after three extractions $(n=3)$. 


\subsection{UHPLC-ITMS Conditions}

A Dionex UltiMate 3000 LC-system (Thermo Fisher Scientific, TF, Dreieich, Germany) connected to an amaZon speed ion trap mass spectrometer (Bruker Daltonik, Bremen, Germany) with an electrospray ionization source were used. Gradient elution was performed on a TF Acclaim RSLC120 C18 column $(100 \mathrm{~mm} \times 2.1 \mathrm{~mm} \times 2.2 \mu \mathrm{m})$ at a column temperature of $45^{\circ} \mathrm{C}$ using eluent $\mathrm{A}$ ( 2 -mM aqueous ammonium formate containing $0.1 \%$ formic acid and $1 \% \mathrm{ACN}$ ) and eluent $\mathrm{B}$ (ACN containing 2-mM ammonium formate, $0.1 \%$ formic acid, and $1 \%$ water). The gradient was programmed as follows: 0 to $1 \mathrm{~min}$ hold $1 \% \mathrm{~B}, 1-4 \mathrm{~min}$ increase to $21 \% \mathrm{~B}, 4-10 \mathrm{~min}$ increase to $33 \% \mathrm{~B}, 10-13 \mathrm{~min}$ increase to $99 \% \mathrm{~B}$, $13-15$ min hold $99 \%$ B, 15 to 15.1 min decrease to $1 \%$ B, and $15.1-16$ min hold $1 \%$ B, each step at a constant flow rate of $0.7 \mathrm{~mL} / \mathrm{min}$. The injection volume was $10 \mu \mathrm{L}$. The mass spectrometer operated in smartMRM mode using a scheduled precursor list (SPL) to generate MS $^{2}$ spectra of scheduled precursors and a mass range from $m / z 70-500$ at $m / z / s ~ 32,500$. SmartMRM mode parameters were programmed as follows: ionization mode, positive; ICC target (determines how many ions should be transferred into the trap), 50,000; max. accumulation time, $50 \mathrm{~ms}$; MS spectra, interval 0.01; averages (determines how many scans will be summed and averaged for one spectrum), 1; SPS for tandem mass spectrometry (MS/MS) acquisition enabled; fast chromatography, not selected; edit parameters, link edit-enabled; and rolling averaging (filtering out short-term fluctuations), No. 1. Source parameters were as follows: capillary voltage, $4500 \mathrm{~V}$; end plate offset, $500 \mathrm{~V}$; nebulizer gas, $80 \mathrm{psi}$; dry gas, $10 \mathrm{~L} / \mathrm{min}$; and dry temperature, $159^{\circ} \mathrm{C}$.

\subsection{Method Validation}

Method validation was performed according to the "Guideline on bioanalytical method validation" of the EMA [30]. Drug identification was based on three criteria: first, the mass of the protonated precursor ion, second, the $\mathrm{MS}^{2}$ spectrum, and third, the $t_{R}$. Data handling was done using Bruker Compass Data Analysis Version 4.4 (Bruker Daltonik). For quantification, peak area ratios of the analyte $\left(\mathrm{m} / z\right.$ of $\mathbf{M}+\mathrm{H}^{+}$are given in Table 2) and the IS diazepam-d5 $\left(\mathrm{M}+\mathrm{H}^{+}\right.$at $\left.m / z 290.11, \mathrm{t}_{\mathrm{R}} 11.30 \mathrm{~min}\right)$ were used. Graphs and structures of analytes were edited using CorelDraw X7 Version 17.0.0.491 (Munich, Germany). Statistical analysis was done using Microsoft Excel 2010 (Redmond, WA, USA) and GraphPad Prism 5.00 (GraphPad Software, La Jolla, CA, USA).

\subsubsection{Ion Suppression and Enhancement of Coeluting Analytes}

Coelution and subsequent ion suppression and enhancement of possible IS was tested for diazepam-d5 $\left(\mathrm{M}+\mathrm{H}^{+}\right.$at $\left.m / z 290.11\right)$ and diazepam $\left(\mathrm{M}+\mathrm{H}^{+}\right.$at $\left.m / z 285.08\right)$, as well as atorvastatin- $\mathrm{d} 5\left(\mathrm{M}+\mathrm{H}^{+}\right.$at $m / z$ 564.29) and atorvastatin $\left(\mathrm{M}+\mathrm{H}^{+}\right.$at $\left.m / z 559.26\right)$. Substances in separate methanolic solutions $(1 \mathrm{mg} / \mathrm{L})$ were injected onto the UHPLC-ITMS system $(n=3)$. The absolute peak areas were compared to the absolute peak areas of a mixture $(n=3)$ containing both substances $(1 \mathrm{mg} / \mathrm{L})$, respectively.

\subsubsection{Selectivity}

Ten plasma samples from different donors were extracted without the addition of IS diazepam-d5, individually analyzed, and evaluated for interferences in order to evaluate whether the current method can differentiate the analytes of interest and IS from endogenous components in the sample. The plasma samples also contained exogenous compounds such as ethanol, sedatives (e.g., midazolam), antidepressants (e.g., mirtazapine), antiepileptics (e.g., valproic acid), analgesics (e.g., metamizole), other antihypertensives (e.g., urapidil), or local anesthetics (e.g., mepivacaine), but none of the cardiovascular drugs were included in the current method development. Selectivity was given if the response was less than $20 \%$ of the LLOQ for the antihypertensive drugs and less than $5 \%$ for the IS diazepam-d5 [30]. 


\subsubsection{Carryover}

For carryover testing, an extract of pooled blank plasma was injected after the highest calibration standard (Cal 6, plasma concentrations of analytes; see Table 1). Carryover should not be greater than $20 \%$ of the LLOQ for the antihypertensive drugs and $5 \%$ for IS diazepam-d5 [30].

\subsubsection{Calibration}

Six calibrator (Cal 1-6) and four QC (LLOQ, low, medium, and high) levels were prepared for each analyte by spiking pooled human blank plasma with predefined analyte concentrations, which are given in Table 1. The LLOQ was defined to be equal to the concentration of Cal 1 and the upper limit of quantification (ULOQ) to the concentration of Cal 6. In total, four different calibration ranges with individual LLOQ and ULOQ levels were defined depending on the analyte. A blank sample (extracted sample without IS diazepam-d5 or analytes) and zero sample (extracted sample with IS diazepam-d5 but without analytes) were prepared. QCs were prepared in duplicates $(n=2)$, and each calibrator, QC, blank, and zero sample was injected and analyzed once. For evaluation, linear and nonlinear regression models were tested, as well as different weighing factors (equal, $1 / x$, and $1 / x^{2}$ ). The AC for the back-calculated concentrations of the calibrator standards were set to $\pm 15 \%$ of the nominal value, except for the LLOQ, for which the back-calculated concentration should be within $\pm 20 \%$ [30]. At least $75 \%$ of the calibration standards must fulfill these criteria, meaning that, in the current case, five out of six calibrator samples must fulfill the criteria.

\subsubsection{Matrix Effect}

According to the EMA guidelines, single plasma samples from six different donors were used instead of the pooled matrix, and two different sample sets were prepared with a low and a high concentration each (for concentrations of low and high QC, see Table 1). The first sample set contained analytes in a pure methanolic solution (neat standard). For the second sample set, blank plasma was extracted and spiked with analytes afterwards.

For each analyte and the IS, the MF was calculated for each lot of the matrix as the ratio of the peak area in the presence of the matrix (sample set 2) and the peak area in the absence of the matrix (sample set 1). Finally, the IS-normalized MF was calculated by dividing the MF of the analyte by the MF of the IS. The CV of the IS-normalized MF calculated from the 6 lots of the matrix should not be greater than $15 \%$.

\subsubsection{Accuracy and Precision}

The closeness of the determined value obtained by the method to the nominal concentration of the analyte is described by the accuracy, which is expressed in percentage. The closeness of repeated individual measures of the analyte is described by the precision that can be expressed as the CV [30]. Freshly prepared QC samples were analyzed against a freshly prepared calibration.

\section{Within-Run Accuracy and Precision}

Within-run accuracy and precision were determined by analyzing five replicates of QCs (LLOQ and low, medium, and high QC; concentration levels given in Table 1) in a single run. The mean concentration should be within $\pm 15 \%$ of the nominal concentration, except for the LLOQ, where it should be within $\pm 20 \%$ of the nominal value, according to the EMA [30]. For within-run precision, CVs were calculated and should be within $15 \%$, except for the LLOQ, where it should be within $20 \%$.

\section{Between-Run Accuracy and Precision}

Between-run accuracy and precision were determined by analyzing five replicates of QCs (LLOQ and low, medium, and high QC; concentration levels given in Table 1) over three runs on three different days. The mean concentration should be within $\pm 15 \%$ of 
the nominal concentration, except for the LLOQ, where it should be within $\pm 20 \%$ of the nominal value, according to the EMA [30]. For between-run precision, CVs were calculated and should not be $>15 \%$, except for the LLOQ, where it should not be $>20 \%$.

\section{Dilution Integrity}

Dilution integrity was tested by spiking pooled human blank plasma with an analyte concentration five times higher than the high QC (for concentrations, see Table 1). After 1:10 dilution with pooled blank plasma, concentrations of medium QC were achieved (concentrations given in Table 1). Samples were extracted and analyzed as described above. Five replicates were prepared $(n=5)$. Accuracy and precision should be within $15 \%$, according to the EMA [30].

\subsubsection{Stability of Stock Solutions}

A methanolic mixture prepared from the stock solutions of the calibrators containing the antihypertensive drugs and the IS diazepam- $\mathrm{d} 5(1 \mathrm{mg} / \mathrm{L}$, each) was analyzed once a week over a period of ten weeks, and CVs of the peak area ratios of the analyte and the IS were calculated. Between the analyses, the mixture was stored at $-20^{\circ} \mathrm{C}$ in an ambercolored vial. CVs not greater than $15 \%$ were defined as acceptable. Ten washing runs injecting an eluent mixture A/B $(1: 1, v / v)$ immediately after analysis prevented carryover.

\subsubsection{Autosampler Stability}

Plasma samples were extracted and analyzed immediately after preparation ( $\mathrm{t} 0)$ and after the appropriate storage condition (t1) against a freshly spiked calibration. Stability of the processed samples (low and high QC; concentrations given in Table $1, n=3$, respectively) in the autosampler was evaluated by reanalyzing the processed samples stored for $20 \mathrm{~h}$ in the autosampler $\left(8^{\circ} \mathrm{C}\right)$. The mean concentration at each concentration level should be within $\pm 15 \%$ of the nominal concentration.

\subsection{Applicability and Calculation of the Dose-Dependent Reference Plasma Concentration Range}

A total of 19 plasma samples of hypertensive patients were extracted and analyzed, as described above. The lower limit of the reference plasma concentration range was represented by $\mathrm{c}_{\min -20 \%}$ (=calculated plasma concentration $24 \mathrm{~h}$ after the latest drug intake minus $20 \%$ ) and the upper limit by the peak plasma concentration $c_{\max }$. The latter was taken from Baselt [33], as well as the corresponding time when the $c_{\max }$ was reached, $t_{\max }$, and the elimination half-life, $t_{1 / 2}$. The shortest given elimination half-life $t_{1 / 2}$ was always used for the calculation of the trough concentration $c_{\min }$. A summary of the pharmacokinetic parameters used for calculation of the reference plasma concentration ranges, as well as an example for such a calculation, are available as Supplementary Materials (Tables S2 and S3, respectively). If the measured plasma concentration was above the ULOQ of the corresponding analyte, the sample was diluted, extracted, and reanalyzed.

\section{Conclusions}

An analytical procedure for the simultaneous quantification of nine cardiovascular drugs based on the LLE of only $100 \mu \mathrm{L}$ of blood plasma and fast UHPLC separation within $12 \mathrm{~min}$, followed by an ITMS analysis, was successfully developed. Validation was successfully performed in accordance with the international guidelines. Limitations exceeded the AC for the matrix effects in the case of amlodipine and its instability. The developed procedure was used for the analysis of 19 plasma samples containing a total of 28 antihypertensive drugs to determine whether a patient can be considered as adherent or not. For this purpose, the dose-dependent reference plasma concentration ranges were calculated using pharmacokinetic parameters. The adherence of two subjects out of 19 could not be assessed $(10 \%)$, due to the measured plasma concentrations and $c_{\min }-20 \%$ below the LLOQ, but 14 were classified as adherent $(75 \%)$ and three as nonadherent $(15 \%)$. In contrast, all $19(100 \%)$ were claimed adherent based on the results of the qualitative urine 
screening due to the presence of the antihypertensive drug and/or metabolites in the urine. The current pilot study demonstrated that the interpretation of plasma concentrations is far more sophisticated and time-consuming than urine screening results, and further data is needed to estimate whether plasma quantification is superior to urine screening in confirming the adherence to antihypertensive medication.

Supplementary Materials: The following are available online: Table S1: Parameters of three different, freshly prepared calibrations acquired during validation procedure (slope, intercept, and deviation of back-calculated concentrations of calibrators. Table S2: Summary of the pharmacokinetic data (elimination half-live $t_{1 / 2}$, peak plasma concentration cmax, and time when cmax was reached, tmax) used for calculating the dose-dependent reference plasma concentration ranges. Table S3: Example calculation of the lower limit $\left(c_{\min -20 \%}\right)$ of the reference plasma concentration range for $10 \mathrm{mg}$ bisoprolol daily.

Author Contributions: Conceptualization, L.W. and M.R.M.; methodology, L.W., A.C.V. and M.R.M.; validation, A.C.V.; formal analysis, A.C.V.; resources, L.L., F.M. and M.R.M.; data curation, L.W., A.C.V. and M.R.M.; writing-original draft preparation, L.W. and A.C.V.; writing-review and editing, L.W., F.M. and M.R.M.; visualization, L.W. and A.C.V.; supervision, L.W. and M.R.M.; and project administration, L.L., F.M. and M.R.M. All authors have read and agreed to the published version of the manuscript.

Funding: This research received no external funding.

Institutional Review Board Statement: This study included patients with hypertension who were referred to the outpatient hypertension unit at the Saarland University Medical Center (Homburg/Saar, Germany). All participating patients provided written informed consent, and the local ethics committee approved the study $(142 / 10)$. Eligible patients were $\geq 18$ years old, had hypertension as defined by the current guidelines, and were prescribed stable antihypertensive therapy for at least two weeks.

Informed Consent Statement: Informed consent was obtained from all subjects involved in the study.

Data Availability Statement: The data can be made available upon reasonable request.

Acknowledgments: The authors would like to thank Birgit Schneider, Armin A. Weber, Gabriele Ulrich, Cathy M. Jacobs, Selina Hemmer, and Fabian Frankenfeld for their support.

Conflicts of Interest: The authors declare no conflict of interest.

Sample Availability: Not applicable.

\section{References}

1. Poulter, N.R.; Prabhakaran, D.; Caulfield, M. Hypertension. Lancet 2015, 386, 801-812. [CrossRef]

2. Ettehad, D.; Emdin, C.A.; Kiran, A.; Anderson, S.G.; Callender, T.; Emberson, J.; Chalmers, J.; Rodgers, A.; Rahimi, K. Blood pressure lowering for prevention of cardiovascular disease and death: A systematic review and meta-analysis. Lancet 2016, 387, 957-967. [CrossRef]

3. Thomopoulos, C.; Parati, G.; Zanchetti, A. Effects of blood pressure lowering on outcome incidence in hypertension: 7. Effects of more vs. less intensive blood pressure lowering and different achieved blood pressure levels—updated overview and meta-analyses of randomized trials. J. Hypertens. 2016, 34, 613-622. [CrossRef] [PubMed]

4. Liu, L.; Wen, Y.; Liu, K.; Sun, L.; Lu, Y.; Yin, Z. Simultaneous determination of a broad range of cardiovascular drugs in plasma with a simple and efficient extraction/clean up procedure and chromatography-mass spectrometry analysis. RSC Adv. 2014, 4, 19629-19639. [CrossRef]

5. WHO. Adherence to Long-Term Therapies: Evidence for Action. 2003. Available online: https://www.who.int/chp/knowledge/ publications/adherence_report/en/ (accessed on 23 February 2021).

6. Burnier, M. Drug adherence in hypertension. Pharmacol. Res. 2017, 125, 142-149. [CrossRef] [PubMed]

7. Overgaauw, N.; Alsma, J.; Brink, A.; Hameli, E.; Bahmany, S.; Peeters, L.E.J.; Van Den Meiracker, A.H.; Schuit, S.C.E.; Koch, B.C.P.; Versmissen, J. Drug nonadherence is a common but often overlooked cause of hypertensive urgency and emergency at the emergency department. J. Hypertens. 2019, 37, 1048-1057. [CrossRef] [PubMed]

8. Saguner, A.M.; Dur, S.; Perrig, M.; Schiemann, U.; Stuck, A.E.; Burgi, U.; Erne, P.; Schoenenberger, A.W. Risk factors promoting hypertensive crises: Evidence from a longitudinal study. Am. J. Hypertens. 2010, 23, 775-780. [CrossRef]

9. Jung, O.; Gechter, J.L.; Wunder, C.; Paulke, A.; Bartel, C.; Geiger, H.; Toennes, S.W. Resistant hypertension? Assessment of adherence by toxicological urine analysis. J. Hypertens. 2013, 31, 766-774. [CrossRef] [PubMed] 
10. Gupta, P.; Patel, P.; Horne, R.; Buchanan, H.; Williams, B.; Tomaszewski, M. How to Screen for Non-Adherence to Antihypertensive Therapy. Curr. Hypertens. Rep. 2016, 18, 89. [CrossRef]

11. Ewen, S.; Meyer, M.R.; Cremers, B.; Laufs, U.; Helfer, A.G.; Linz, D.; Kindermann, I.; Ukena, C.; Burnier, M.; Wagenpfeil, S.; et al. Blood pressure reductions following catheter-based renal denervation are not related to improvements in adherence to antihypertensive drugs measured by urine/plasma toxicological analysis. Clin. Res. Cardiol. 2015, 104, 1097-1105. [CrossRef]

12. Kunz, M.; Lauder, L.; Ewen, S.; Bohm, M.; Mahfoud, F. The Current Status of Devices for the Treatment of Resistant Hypertension. Am. J. Hypertens. 2020, 33, 10-18. [CrossRef]

13. Vitolins, M.Z.; Rand, C.S.; Rapp, S.R.; Ribisl, P.M.; Sevick, M.A. Measuring adherence to behavioral and medical interventions. Control. Clin. Trials 2000, 21, 188S-194S. [CrossRef]

14. Norell, S.E. Accuracy of patient interviews and estimates by clinical staff in determining medication compliance. Soc. Sci. Med. E 1981, 15, 57-61. [CrossRef]

15. Richter, L.H.J.; Jacobs, C.M.; Mahfoud, F.; Kindermann, I.; Bohm, M.; Meyer, M.R. Development and application of a LCHRMS/MS method for analyzing antihypertensive drugs in oral fluid for monitoring drug adherence. Anal. Chim. Acta 2019, 1070, 69-79. [CrossRef] [PubMed]

16. Tomaszewski, M.; White, C.; Patel, P.; Masca, N.; Damani, R.; Hepworth, J.; Samani, N.J.; Gupta, P.; Madira, W.; Stanley, A.; et al. High rates of non-adherence to antihypertensive treatment revealed by high-performance liquid chromatography-tandem mass spectrometry (HP LC-MS/MS) urine analysis. Heart 2014. [CrossRef] [PubMed]

17. Helfer, A.G.; Michely, J.A.; Weber, A.A.; Meyer, M.R.; Maurer, H.H. Orbitrap technology for comprehensive metabolite-based liquid chromatographic-high resolution-tandem mass spectrometric urine drug screening-exemplified for cardiovascular drugs. Anal. Chim. Acta 2015, 891, 221-233. [CrossRef] [PubMed]

18. Gupta, P.; Patel, P.; Strauch, B.; Lai, F.Y.; Akbarov, A.; Gulsin, G.S.; Beech, A.; Maresova, V.; Topham, P.S.; Stanley, A.; et al. Biochemical Screening for Nonadherence Is Associated With Blood Pressure Reduction and Improvement in Adherence. Hypertension 2017, 70, 1042-1048. [CrossRef] [PubMed]

19. Hamdidouche, I.; Jullien, V.; Boutouyrie, P.; Billaud, E.; Azizi, M.; Laurent, S. Routine urinary detection of antihypertensive drugs for systematic evaluation of adherence to treatment in hypertensive patients. J. Hypertens. 2017, 35, 1891-1898. [CrossRef] [PubMed]

20. Helfer, A.G.; Michely, J.A.; Weber, A.A.; Meyer, M.R.; Maurer, H.H. Liquid chromatography-high resolution-tandem mass spectrometry using Orbitrap technology for comprehensive screening to detect drugs and their metabolites in blood plasma. Anal. Chim. Acta 2017, 965, 83-95. [CrossRef]

21. Peters, F.T. Recent developments in urinalysis of metabolites of new psychoactive substances using LC-MS. Bioanalysis 2014, 6, 2083-2107. [CrossRef]

22. Punt, A.M.; Stienstra, N.A.; van Kleef, M.E.A.; Lafeber, M.; Spiering, W.; Blankestijn, P.J.; Bots, M.L.; van Maarseveen, E.M. Screening of cardiovascular agents in plasma with LC-MS/MS: A valuable tool for objective drug adherence assessment. J. Chromatogr. B Anal. Technol. Biomed. Life Sci. 2019, 1121, 103-110. [CrossRef]

23. Lum, G.; Mushlin, B. Urine Drug Testing: Approaches to Screening and Confirmation Testing. Lab. Med. 2004, 35, 368-373. [CrossRef]

24. Ritscher, S.; Hoyer, M.; Georges, C.; Wunder, C.; Wallemacq, P.; Persu, A.; Obermuller, N.; Toennes, S.W. Benefit of serum drug monitoring complementing urine analysis to assess adherence to antihypertensive drugs in first-line therapy. PLoS ONE 2020, 15, e0237383. [CrossRef] [PubMed]

25. Ritscher, S.; Hoyer, M.; Wunder, C.; Obermuller, N.; Toennes, S.W. Evaluation of the dose-related concentration approach in therapeutic drug monitoring of diuretics and beta-blockers-drug classes with low adherence in antihypertensive therapy. Sci. Rep. 2019, 9, 15652. [CrossRef] [PubMed]

26. van der Nagel, B.C.H.; Versmissen, J.; Bahmany, S.; van Gelder, T.; Koch, B.C.P. High-throughput quantification of 8 antihypertensive drugs and active metabolites in human plasma using UPLC-MS/MS. J. Chromatogr. B Anal. Technol. Biomed. Life Sci. 2017, 1060, 367-373. [CrossRef] [PubMed]

27. De Nicolo, A.; Avataneo, V.; Rabbia, F.; Bonifacio, G.; Cusato, J.; Tomasello, C.; Perlo, E.; Mulatero, P.; Veglio, F.; Di Perri, G.; et al. UHPLC-MS/MS method with protein precipitation extraction for the simultaneous quantification of ten antihypertensive drugs in human plasma from resistant hypertensive patients. J. Pharm. Biomed. Anal. 2016, 129, 535-541. [CrossRef]

28. Schwabe, U.; Paffrath, D.; Ludwig, W.-D.; Klauber, J. Arzneiverordnungs-Report 2018; Springer: Heidelberg, Germany, 2018.

29. Schwabe, U.; Paffrath, D.; Ludwig, W.-D.; Klauber, J. Arzneiverordnungs-Report 2019; Springer: Heidelberg, Germany, 2019.

30. EMA. Guideline on Bioanalytical Method Validation, 2015th ed.; European Medicines Agency: London, UK, 2011; Volume EMEA/CHMP/EWP/192217/2009 Rev.1 Corr.2.

31. Caspar, A.T.; Meyer, M.R.; Maurer, H.H. Blood plasma level determination using an automated LC-MS(n) screening system and electronically stored calibrations exemplified for 22 drugs and two active metabolites often requested in emergency toxicology. Drug Test. Anal. 2018. [CrossRef]

32. Schulz, M.; Schmoldt, A.; Andresen-Streichert, H.; Iwersen-Bergmann, S. Revisited: Therapeutic and toxic blood concentrations of more than 1100 drugs and other xenobiotics. Crit. Care 2020, 24, 195. [CrossRef]

33. Baselt, R.C. Disposition of Toxic Drugs and Chemicals in Man, 11th ed.; Biomedical Publications: Seal Beach, CA, USA, 2017. 
34. Avataneo, V.; De Nicolo, A.; Rabbia, F.; Sciandra, M.; Tosello, F.; Cusato, J.; Perlo, E.; Fatiguso, G.; Allegra, S.; Favata, F.; et al. A simple UHPLC-PDA method with a fast dilute-and-shot sample preparation for the quantification of canrenone and its prodrug spironolactone in human urine samples. J. Pharmacol. Toxicol. Methods 2018, 94, 29-35. [CrossRef]

35. Remane, D.; Meyer, M.R.; Wissenbach, D.K.; Maurer, H.H. Ion suppression and enhancement effects of co-eluting analytes in multi-analyte approaches: Systematic investigation using ultra-high-performance liquid chromatography/mass spectrometry with atmospheric-pressure chemical ionization or electrospray ionization. Rapid Commun. Mass Spectrom. 2010, 24, 3103-3108. [CrossRef]

36. GTFCh. Recommendations of criteria for development and validation of analytical methods for estimating concentrations of drugs in blood to be used in 24/7 clinical toxicology. Toxichem Krimtech 2018, 85, 35.

37. Schulz, M.; Iwersen-Bergmann, S.; Andresen, H.; Schmoldt, A. Therapeutic and toxic blood concentrations of nearly 1000 drugs and other xenobiotics. Crit. Care 2012, 16, R136. [CrossRef] [PubMed]

38. Rognstad, S.; Soraas, C.L.; Bergland, O.U.; Hoieggen, A.; Strommen, M.; Helland, A.; Opdal, M.S. Establishing Serum Reference Ranges for Antihypertensive Drugs. Ther. Drug Monit. 2021, 43, 116-125. [CrossRef] [PubMed]

39. Hiemke, C.; Bergemann, N.; Clement, H.W.; Conca, A.; Deckert, J.; Domschke, K.; Eckermann, G.; Egberts, K.; Gerlach, M.; Greiner, C.; et al. Consensus Guidelines for Therapeutic Drug Monitoring in Neuropsychopharmacology: Update 2017. Pharmacopsychiatry 2017, 15, 1645-3265. [CrossRef] 